\title{
Successful treatment of xanthogranulomatous pyelonephritis in an elderly lady with percutaneous drainage
}

\author{
Tan Jih Huei ${ }^{1}$, Henry Tan Chor Lip ${ }^{1}$, Shamsuddin Omar $^{2}$ \\ ${ }^{1}$ Department of General Surgery, Hospital Sultanah Aminah, Johor Bahru, Malaysia. \\ ${ }^{2}$ Department of Urology, Hospital Sultanah Aminah, Johor Bahru, Malaysia.
}

\section{Keywords}

Xanthogranulomatous pyelonephritis; renal cell carcinoma; renal calculi

\section{Introduction}

Xanthogranulomatous Pyelonephritis (XGP) is a severe form of pyelonephritis characterized by destructive changes to renal parenchyma due to chronic inflammation by bacterial infection. In general a significant number of patients undergo a nephrectomy as these lesions mimic renal tumors. If the diagnosis of XGP is suspected, a partial nephrectomy may be attempted. When the infection is extensive, a nephrectomy together with debridement of surrounding tissue may be needed. Less commonly, the condition can be treated with intravenous antibiotic alone or percutaneous drainage. Here, we report an elderly lady who presented with painful right lumbar mass. Magnetic Resonance Urography revealed a large renal collection with mixed density. The lady recovered following percutaneous drainage.

\section{Case presentation}

An 80 years old Chinese lady presented with right lumbar pain and mass for 2 weeks. It was associated with lethargy and appetite loss. She had a history of right renal stone which had been treated with open surgery 15 years ago. She had chronic kidney disease with baseline creatinine of $210 \mathrm{umol} / \mathrm{L}$. She had consumed over the counter analgesic whenever she had right lumbar pain. She had no diabetes mellitus. Dextrostix was $5.9 \mathrm{mmol} / \mathrm{L}$. Her blood pressure was $150 / 92 \mathrm{mmHg}$ and pulse rate was 116 beats per min. A right lumbar mass was felt. It was non-tender and measuring $20 \times 15 \mathrm{~cm}$ in size.

Her urine biochemistry revealed cloudy urine with negative nitrite and leucocytes of $2+$. Urine culture and sensitivity had a negative yield. White cell counts were $13.2 \times 10^{\wedge} 9 / \mathrm{L}$, haemoglobin $84 \mathrm{~g} / \mathrm{L}$, platelet $77 \times 10^{\wedge} 9 / \mathrm{L}$, urea $24.5 \mathrm{mmol} / \mathrm{L}$ and creatinine $480 \mathrm{umol} / \mathrm{L}$. Her blood gas revealed compensated metabolic acidosis with pH 7.377, pCO2 25.8, base deficit of -9.4 .

Correspondence: Tan Jih Huei

E-mail: huei_87@hotmail.com

Received: 09-05-2018 Accepted: 21-07-2018

(iD) http://orcid.org/0000-0001-9833-5164

DOI: http://doi.org/10.4038/sljs.v36i3.8526

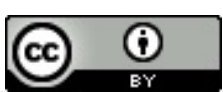

Kidney ureter bladder radiography revealed right lumbar homogenous density with a large radiopaque stone. Ultrasound (USG) revealed grossly enlarged right kidney with heterogeneous echogenicity and multiple renal calculi. Non contrasted computed tomography of kidney, ureter and bladder (CT KUB) revealed diffusely enlarged right kidney measuring $14.3 \mathrm{~cm} \times 10.7 \mathrm{~cm} \times 10.5 \mathrm{~cm}$. The kidney contained cystic and complex solid components. There were multiple renal calculi largest $4.9 \mathrm{~cm}$ in its diameter (Figure 1).

In view of suspicious features on CT KUB and USG, the diagnosis of renal tumour was not able to be excluded.

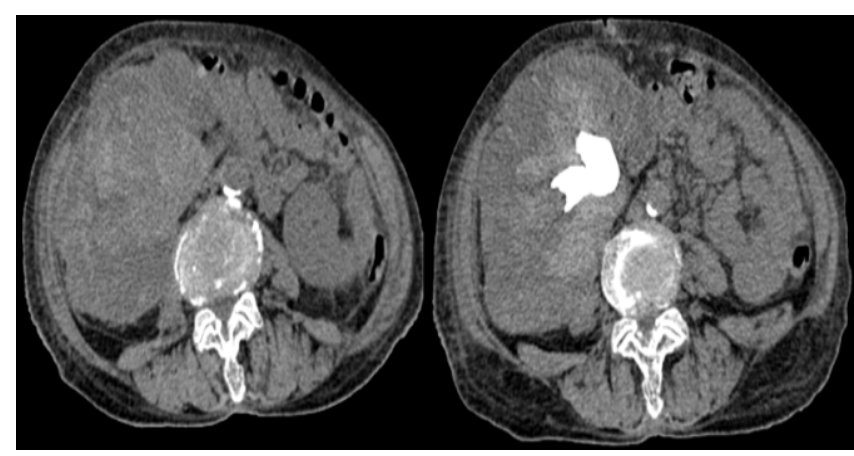

Figure 1. Plain CT axial view showing hypodensity area within the right renal mass

Therefore, magnetic resonance (MR) kidney, ureter \& bladder was done due to impaired renal function. A large right renal collection with mixed density was identified. The centre and anterior portion of the lesion showed hypointense signal on T1 weighted imaging suggesting a fluid collection (Figure 2).

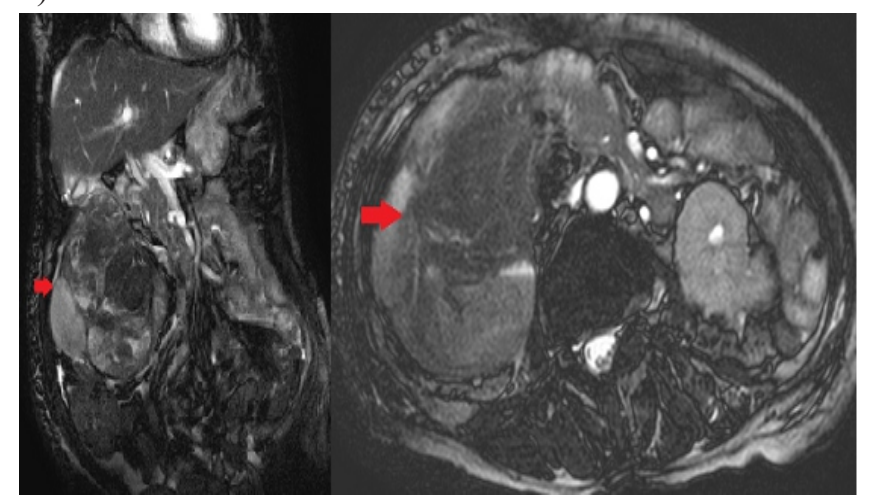

Figure 2. MRI showing right renal mass lesion (red arrow) in coronal and axial view 
Based on the collective findings of all the imaging results, a diagnosis of xanthogranulomatous pyelonephritis was made. A percutaneous drain was inserted into the right renal collection.. One litre of haemopurulent fluid was drained on aspiration. Subsequently, it drained about hundred $\mathrm{ml}$ per day for 3 days. Her pain reduced and appetite was regained following the procedure.

\section{Discussion}

Xanthogranulomatous pyelonephritis is an uncommon and severe form of pyelonephritis with destructive changes involving renal parenchyma[1]. The entity commonly involve individual of the age of 45 to 55 years with female predominance. It present in 2 forms, either diffuse or local type. Clinical presentation and radiological findings may mimic a neoplastic aetiology $[1,2]$. In the current case, we faced a similar challenge to differentiate the cause of the renal mass from a renal cell carcinoma.

Computed tomography (CT) scan is regarded as the main modality to diagnose the condition $[1,3]$.The CT findings may include hydronephrosis (90.9\%), renal calculus (72.7\%), pyonephrosis $(45.5 \%)$, intraparenchymatous collection (45.5\%), cortical renal atrophy (45.5\%), nonfunctioning kidney $(36.4 \%)$, abscess $(36.4 \%)$, or perinephric fat accumulation (18.2\%) [1].Hydronephrosis, renal calculus, and intraparenchymatous collections were present in the CT scan of the case. However, the non-contrasted nature of the scan limited the ability to differentiate from a malignant etiology.

Magnetic resonance imaging (MRI) gives extra information in addition to non- contrasted CT. As XGP presents like a pseudo-cystic mass, it generates low-intensity signal on T1weighted images and high-intensity signal on T2-weighted images. This was observed in this case. Histologically in $\mathrm{XGP}$, the renal parenchyma is replaced by lipid-laden foamy macrophages in combination with an inflammatory granuloma and a lymphoplasmacytic infiltrate. MRI is sensitive to identify the lipid laden foamy macrophages as high-intensity signal on spin-echo, T1-weighted images. However, angiomyolipoma (AML), retroperitoneal liposarcoma, and renal cell carcinoma (RCC) occasionally contain fatty tissue. The limitation of MRI is often described in differentiating RCC from two common benign entities which are minimal-fat AML and oncocytoma. AML demonstrated decreased T2 signal intensity, increased T1 signal intensity, high signal loss on opposed-phase images, and high ratio in signal intensity between early and delayed post contrast image. This overlaps with features of RCC on MRI. Similarly in the case of oncytoma, central scarring with areas of necrosis and local aggressive behaviour of perirenal fat invasion or renal vein branch invasion can mimic a RCC. Therefore, this confirmation of the renal mass pathology can only be relied on biopsy [4].

In this case, the diagnosis was made after consideration of the clinical history and combination of several radiological imaging modalities. The collective findings such as intraparenchymatous renal collection and renal calculi prompted an infectious etiology. The immediate drainage of the lesion successfully relieved the symptoms of the elderly lady in short term. In conclusion, diagnosing XGP prior to any intervention remains difficult. The combination of $\mathrm{CT}$ and MRI may aid in differentiating from a renal cell carcinoma. Percutaneous drainage is essential measure to relieve acute symptoms. A subsequent nephrectomy maybe required for complete cure of this disease.

All authors disclose no conflict of interest. The study was conducted in accordance with the ethical standards of the relevant institutional or national ethics committee and the Helsinki Declaration of 1975, as revised in 2000 .

\section{References}

1. . Li L, Parwani AV. Xanthogranulomatous pyelonephritis. Arch Pathol Lab Med. 2011 May;135(5):671-4. doi: 10.1043/2009-0769-RSR.1.

2. Loffroy R, Guiu B, Watfa J, Michel F, Cercueil JP, Krausé D. Xanthogranulomatous pyelonephritis in adults: clinical and radiological findings in diffuse and focal forms. Clin Radiol. 2007 Sep;62(9):884-90. https://doi.org/10.1016/j.crad.2007.04.008

3. Said Conti V, Azzopardi C, Fearne C, Cuckow P. Xanthogranulomatous pyelonephritis: the case of a perplexing kidney. BMJ Case Rep [Internet]. 2014 Sep 19;2014. Available from: http://www.ncbi.nlm.nih.gov/pmc/articles/PMC4170517/

4. Campbell N, Rosenkrantz AB, Pedrosa I. MRI Phenotype in Renal Cancer : is it clinically relevant? Top Magn Reson Imaging. 2014Apr;23(2):95-115.

doi: 10.1097/RMR.0000000000000019.

\section{Learning Points:}

- Xanthogranulomatous pyelonephritis can mimic renal cell carcinoma. Combination of clinical history and few imaging modalities can be useful to differentiate from renal cell carcinoma.

- The disease (XGP) is usually treated with nephrectomy. However, selected patients may benefit from initial percutaneous drainage with the intent of avoiding nephrectomy.

- MRI is an alternative imaging modality whenever doubt exists with routine imaging with radiography and computed tomography scan. 